\title{
¿Puede un aumento en el ingreso generar trampas de pobreza? Recursos naturales y trabajo infantil*
}

Recibido: 24 de febrero de 2016 - Aceptado: 25 de agosto de 2016

Doi: dx.doi.org/10.12804/revistas.urosario.edu.co/economia/a.5242

Hernando Zuleta ${ }^{+}$

Juliana Roa ${ }^{\ddagger}$

\section{Resumen}

Este artículo presenta una aproximación teórica a la relación entre el precio de los recursos naturales y las trampas de pobreza para el caso de un país productor de minerales. Partiendo de un modelo de generaciones traslapadas donde las decisiones de ahorro, inversión en capital humano, fertilidad y trabajo infantil son endógenas, se identifican las condiciones en las cuales un aumento en el precio de los recursos naturales genera un incremento en el trabajo infantil y una caída en el crecimiento económico. Del mismo modo se identifican las condiciones en las que existe una tasa de crecimiento sostenida. Se encuentran cuatro resultados centrales: 1) Altos precios de los recursos naturales pueden generar trampas de pobreza. 2) Dado el precio de los recursos naturales, entre mayor sea la productividad del trabajo en el sector educativo menor es la probabilidad de existencia de trampas de pobreza. 3) Cuando existe trabajo infantil, la tasa de crecimiento del capital humano depende negativamente del precio de los recursos naturales. 4) La existencia de trabajo infantil puede llevar a caídas en el capital humano y en el capital físico.

Clasificación JeL: E24, K42, O1, O15, O17, O4, R2.

Palabras clave: trampas de pobreza, minería, trabajo infantil, recursos naturales, educación, crecimiento.

* Agradecemos a Emilio Depetris, Óscar Ávila, Rafael Santos, Harold Villalba y a un evaluador anónimo por sus comentarios y sugerencias.

+ Departamento de Economía, Universidad de los Andes, Carrera 1 N 18 A-70 Bloque W, piso 9 (Bogotá, Colombia). Teléfono: (+571) 3394949 ext. 2464. Correo electrónico: h.zuleta@uniandes.edu.co

$\ddagger$ Departamento de Economía, Universidad de los Andes, Carrera 1 N$^{\circ} 18 \mathrm{~A}-70$ Bloque W, piso 8 (Bogotá, Colombia). Correo electrónico: j.roa43@uniandes.edu.co

Para citar este artículo: Zuleta, H., \& Roa, J. (enero-junio, 2016). ¿Puede un aumento en el ingreso generar trampas de pobreza? Recursos naturales y trabajo infantil. Revista de Economía del Rosario, 19(1), 85-115. Doi: dx.doi.org/10.12804/revistas.urosario.edu.co/economia/a.5242 


\title{
Can a positive income shock generate a poverty trap? Commodities and child labor
}

\begin{abstract}
We present a theoretical model in order to study the causal relation between commodity prices and poverty traps. Using an overlapping generation's framework where savings, education, fertility and child labor are endogenous variables we find four central results: 1) High commodity prices may generate poverty traps. 2) Given commodity prices, high labor productivity in the production of human capital reduces the likelihood of a poverty trap. 3) When there is child labor, the growth rate of human capital negatively depends on the commodity prices. 4) Child labor may lead to a decrease in both physical and human capital.
\end{abstract}

JEL classification: E24, K42, O1, O15, O17, O4, R2.

Keywords: poverty traps, mining, child labor, natural resources, education, savings, economic growth.

\section{Pode um aumento no ingresso gerar armadilhas de pobreza? Recursos naturais e trabalho infantil}

\author{
Resumo
}

Este artigo apresenta uma aproximação teórica à relação entre o preço dos recursos naturais e as armadilhas da pobreza para o caso de um país produtor de minerais. Partindo de um modelo de gerações sobrepostas onde as decisões de poupança, investimento em capital humano, fertilidade e trabalho infantil são endógenas, se identificam as condições nas quais um aumento no preço dos recursos naturais gera um incremento no trabalho infantil e uma caída no crescimento econômico. Do mesmo modo identificam-se as condições nas que existe uma taxa de crescimento sustentada. Encontram-se quatro resultados centrais: 1) Altos preços dos recursos naturais podem gerar armadilhas de pobreza. 2) Dado o preço dos recursos naturais, entre maior seja a produtividade do trabalho no setor educativo menor é a probabilidade de existência de armadilhas de pobreza. 3) Quando existe trabalho infantil, a taxa de crescimento do capital humano depende negativamente do preço dos recursos naturais. 4) A existência de trabalho infantil pode levar a caídas no capital humano e no capital físico.

Classificação JEL: E24, K42, O1, O15, O17, O4, R2.

Palavras-chave: Armadilhas de pobreza, mineração, trabalho infantil, recursos naturais, educação, crescimento. 


\section{Introducción}

Los proyectos de minería son los últimos proyectos que un legislador prudente, que desee incrementar la riqueza de su nación, elegiría

para incentivar. ADAM SMITH (1776)

El trabajo infantil a menudo está asociado con explotación y jornadas de trabajo extensas. En estas condiciones, los niños trabajadores suelen padecer problemas de salud y retrasos en el desarrollo. Así mismo, el trabajo de la niñez es uno de los determinantes del atraso escolar (Pinzón-Rondón et al., 2006). En este orden de ideas, el trabajo infantil afecta el bienestar de los niños, la dinámica de acumulación de capital humano y, por lo tanto, la tasa de crecimiento de la economía (Ishikawa, 1975; Parsons, 1977).

Ahora, el costo de oportunidad de enviar a los niños al colegio es el ingreso derivado del trabajo infantil y la inversión en capital humano tiene retornos de largo plazo, mientras que el trabajo infantil produce ingresos en el corto plazo. Así, los hogares más impacientes o con necesidades de corto plazo más apremiantes escogen el trabajo infantil.

Por otro lado, dado que el ingreso que aportan los niños al hogar depende positivamente del precio del bien producido con ayuda del trabajo infantil, cuando crece el precio de estos bienes aumenta también el costo de oportunidad de enviar los niños a estudiar y, por lo tanto, puede incrementar el trabajo infantil. Debido a que el trabajo realizado por los menores de edad es trabajo no calificado, aumentos en el precio de bienes cuya producción es intensiva en trabajo no calificado pueden llevar a incrementos en el trabajo infantil. Este es el caso de la minería tradicional y algunos productos agrícolas (recursos naturales).

Si los padres son altruistas, entre mayor es su salario mayor es la inversión en educación de los hijos. Así, cuando los aumentos en el precio de los recursos naturales están acompañados de incrementos proporcionales en los salarios de los adultos, no hay efectos sobre el trabajo infantil.

Por último, cuando las condiciones son tales que las familias deciden enviar a sus hijos a trabajar, los aumentos en el retorno al trabajo infantil son también aumentos en el retorno de la fertilidad. Por este motivo, los incrementos en el precio de los recursos naturales generan también aumento en la tasa de crecimiento poblacional, lo cual, a su vez, reduce el crecimiento del capital por trabajador.

Estas interacciones se formalizan en un modelo teórico que permite analizar los efectos de un aumento en el precio de los recursos naturales sobre 
las decisiones de trabajo infantil, ahorro y tasa de fertilidad. Dado que la dinámica del crecimiento económico depende de la acumulación de capital físico y capital humano, las relaciones mencionadas pueden explicar la forma en la que un boom de recursos naturales puede generar trampas de pobreza.

Los resultados del modelo sugieren que, dado el salario de los padres, aumentos en el precio de los recursos naturales incrementan los incentivos de los padres de enviar a sus hijos a trabajar y, por lo tanto, pueden generar trampas de pobreza. Ahora, entre mayor es la productividad del trabajo en el sector educativo, mayor es el costo de oportunidad del trabajo infantil $\mathrm{y}$, por lo tanto, menor es la probabilidad de que existan trampas de pobreza. Teniendo en cuenta estos dos resultados, la asistencia escolar de los niños se ve afectada tanto por los precios de los recursos naturales como por la calidad educativa. En caso de precios altos de recursos naturales y baja productividad en la educación, la asistencia escolar es baja y, por ende, las posibilidades de movilidad social de la familia son reducidas. ${ }^{1}$

El caso de la minería informal en Colombia sirve de motivación para el modelo teórico. Las características de la población que habita las zonas de minería informal, los bajos salarios de mercado y la incidencia del trabajo infantil parecen ser determinantes del estancamiento económico.

Este artículo está compuesto de cinco secciones, de las cuales esta introducción es la primera. En la segunda sección, se presenta una revisión de la literatura. En la sección 3, se describen los efectos de los aumentos en el precio de los recursos naturales y se expone, a manera de ejemplo, el caso de la minería artesanal en Colombia. En la sección 4, se muestra el modelo teórico. La sección 5 concluye.

\section{Revisión de la literatura}

El modelo que se presenta está relacionado con tres líneas de la literatura económica: trabajo infantil, natalidad y enfermedad holandesa. ${ }^{2}$

1 Siguiendo la tradición de sus ancestros, los hijos aprenden del padre o la madre el único oficio que han conocido en su historia familiar, y, a su vez, el padre o la madre tiende a consolidar el valor formativo que culturalmente atribuye al oficio de la minería - $\mathrm{Mi}$ nercol, OIT-IPEC. (2001a). Las niñas y los niños que trabajan en la minería artesanal colombiana. Diagnóstico sociocultural, económico y legislativo (1ª ed.). Bogotá-.

2 Ver Becker (1994), Becker y Tomes (1994), Van der Ploeg (2011), Kraay y Mckenzie (2014), Edmonds (2004) y Santos (2014) para una revisión completa de la literatura. 
Varios autores han estudiado la relación entre ingresos del hogar, educación y trabajo infantil. Psacharopoulos (1996) encuentra que, en promedio, aquellos niños que trabajan ven reducida su tasa de escolaridad en dos años, y experimentan un aumento en la probabilidad de repetir un año escolar, para el caso de Bolivia y Venezuela. En la misma dirección, Bezerra, Kassouf y Kuenning (2009) utilizan medidas directas del rendimiento en pruebas académicas de matemáticas y portugués, y reportan una relación negativa entre trabajo infantil y rendimiento escolar en Brasil.

La relación entre el número de hijos en el hogar y el trabajo infantil también ha sido estudiada. Varios autores encuentran una relación positiva entre el número de hijos por hogar y el trabajo infantil. Patrinos y Psacharopoulos (1997) abordan este tema para el caso de Perú y observan que, en los hogares con mayor número de niños de corta edad, la probabilidad del trabajo infantil es mayor. En el caso de Colombia, Gómez, Giraldo, Ramírez y Aparicio (2013) reportan que, para los hogares con proporción alta de niños menores a cinco años, hay un aumento en la probabilidad de trabajo infantil. ${ }^{3}$ Finalmente, Heckman y Raut (2013) describen que la inversión en preescolar aumenta significativamente las habilidades cognitivas y no cognitivas de los niños, lo cual incrementa las ganancias del hogar a futuro y mejora los resultados escolares. Paralelamente, los autores demuestran que las habilidades no cognitivas son determinantes esenciales tanto para las ganancias futuras como para el aprendizaje.

En la línea de tasas de natalidad y desarrollo, la evidencia indica que la formación de capital humano es determinante para la transición demográfica y también genera las condiciones para un crecimiento económico sostenido (Galor \& Moav, 2004; Galor, 2005). En primer lugar, la reducción de la tasa de fecundidad genera una caída en el número de integrantes por familia, lo cual tiene un efecto positivo, ya que incrementa la producción per capita (Galor \& Zang, 1997). En segundo lugar, caídas en la fecundidad aumentan la acumulación de capital humano dado (Galor \& Weil, 1999). Por último, caídas en la tasa de fecundidad afectan la estructura de edad en la población, reduciendo la tasa de dependencia e impulsando el PIB per capita (Doepke, 2004).

Finalmente, la literatura acerca de la enfermedad holandesa parte de una regularidad empírica: los países con recursos naturales abundantes crecen a tasas inferiores a las de los demás países. Este fenómeno se conoce también

3 Estos estudios demuestran que hay una correlación positiva entre el tamaño del hogar y el trabajo infantil. No obstante, Rosati y Rossi (2003) encuentran, para el caso de Nicaragua y Pakistán, que, entre más hijos haya en el hogar, el número de horas de trabajo infantil disminuye y es más probable que los manden al colegio. 
como la maldición de los recursos naturales. ${ }^{4}$ Igualmente, se observa que aumentos en el precio de recursos naturales abundantes generan síntomas de la enfermedad holandesa.

En este trabajo se estudia el efecto de aumentos en el precio de los recursos naturales en las decisiones familiares. En particular, partiendo de un modelo de generaciones traslapadas, se considera el caso de familias residentes en regiones abundantes en bienes cuya producción se realiza de manera informal. Las familias pueden enviar a sus hijos al colegio o a trabajar en la producción del recurso abundante. Cada familia toma el salario de los adultos, el precio del recurso y la productividad escolar como dados, y toma decisiones sobre ahorro, número de hijos y trabajo infantil.

Los supuestos y la estructura del modelo que presentamos son consistentes con la evidencia empírica y construyen sobre el aparato teórico desarrollado por Galor y colegas. La principal contribución del trabajo es aportar un modelo teórico que formaliza las relaciones entre la variación de precios de los recursos naturales, el trabajo infantil, la tasa de natalidad y el crecimiento económico. Así mismo, a partir de los resultados del modelo se derivan recomendaciones de política pública: en primer lugar, la productividad en la provisión de educación tiene implicaciones de corto plazo sobre el trabajo infantil y de largo plazo sobre la senda de crecimiento económico. En segundo lugar, la relación entre las variaciones en los precios de los recursos naturales (minería en particular) y las decisiones de trabajo infantil llaman la atención acerca de la necesidad de fortalecer o rediseñar programas sociales en regiones donde el trabajo infantil es común.

\section{Recursos naturales y trabajo infantil}

De acuerdo con Goldin y Parsons (1981), la presencia de industrias con una alta demanda de trabajo infantil reduce el bienestar económico de las siguientes generaciones. Para el caso de los Estados Unidos, el aumento en el ingreso del hogar generado por trabajo infantil es absorbido por un incremento en el consumo. Es decir, la ganancia económica derivada del trabajo infantil se ve opacada por los efectos de un aumento en el consumo. ${ }^{5}$

4 Algunos artículos destacados en la literatura sobre enfermedad holandesa son: Corden (1984), Auty (1993), Sachs y Warner (1995, 1999), Davis (1995), Barder (2006), Ismail (2010) y Keniston y Allcott (2014).

5 No obstante, si el sector que experimenta cambios en precios emplea mayoritariamente adultos, el efecto puede ser contrario. Cogneau y Jedwab (2007) encuentran para el 
En Colombia, la minería tradicional ha logrado trascender a lo largo del tiempo. Las primeras comunidades que habitaron las zonas de minearía artesanal comenzaron esta tradición. ${ }^{6}$ Infortunadamente, la especialización en minería artesanal representa una barrera hacia la movilidad social.

Las comunidades que se dedican a la minería tienen una baja valoración de la educación. El bajo nivel educativo de los adultos, junto a las bajas tasas de inscripción en escuelas, contribuye a la creencia de que el trabajo infantil ayuda a la formación y, por este motivo, la educación no se concibe como un derecho fundamental. Del mismo modo, la minería tradicional suele desarrollarse de manera informal, genera aumentos en la deserción escolar e implica riesgos para la salud y la vida de los trabajadores.

La figura 1 ilustra la relación entre trabajo infantil y asistencia escolar para el caso colombiano. Para el rango total de edades entre 7 y 17 años de edad, quienes trabajan presentan niveles inferiores de asistencia escolar que quienes no trabajan. Además, esta diferencia crece con la edad de los menores. Esto último se explica porque la fuerza y la capacidad de trabajo suele aumentar con la edad y, por lo tanto, el costo de oportunidad de estudiar se incrementa también.

La tabla 1 presenta la distribución de minería ilegal por departamentos colombianos. Los datos están elaborados a partir de las principales unidades del esquema de división política y geográfica y de la información registrada en el censo minero nacional 2009-2011. Existen 14357 minas, de las cuales 9040 no poseen un título minero, esto representa el 62,9\% del total de la actividad minera en el país.

En 23 de los 32 departamentos de Colombia se practica minería ilegal, siendo los departamentos de Antioquia, Bolívar, Boyacá, Cundinamarca y Santander las cinco zonas donde más se presenta este fenómeno. El 35,1 \% de los municipios está afectado por la minería ilegal.

La tabla 2 presenta la información de trabajo infantil por departamento. Algunos departamentos no reportan casos de trabajo infantil en minería, pero los departamentos con más minas ilegales son los que más trabajo infantil reportan.

caso de Costa de Marfil que una disminución en los precios de la cocoa reduce el ingreso del hogar y, debido a esto, los padres se ven obligados a enviar a sus hijos a trabajar para poder recuperar ese ingreso perdido a causa de la disminución de precios. Como consecuencia, se genera una caída del $3 \%$ en la probabilidad de asistencia escolar.

6 Los hogares, al percibir una creciente demanda de trabajo, contemplan la posibilidad de utilizar a sus hijos como una fuente extra de ingresos al enviarlos a trabajar en las minas y los hijos aprenden de sus padres la única labor conocida en su historia familiar (Melguizo, 2004). 


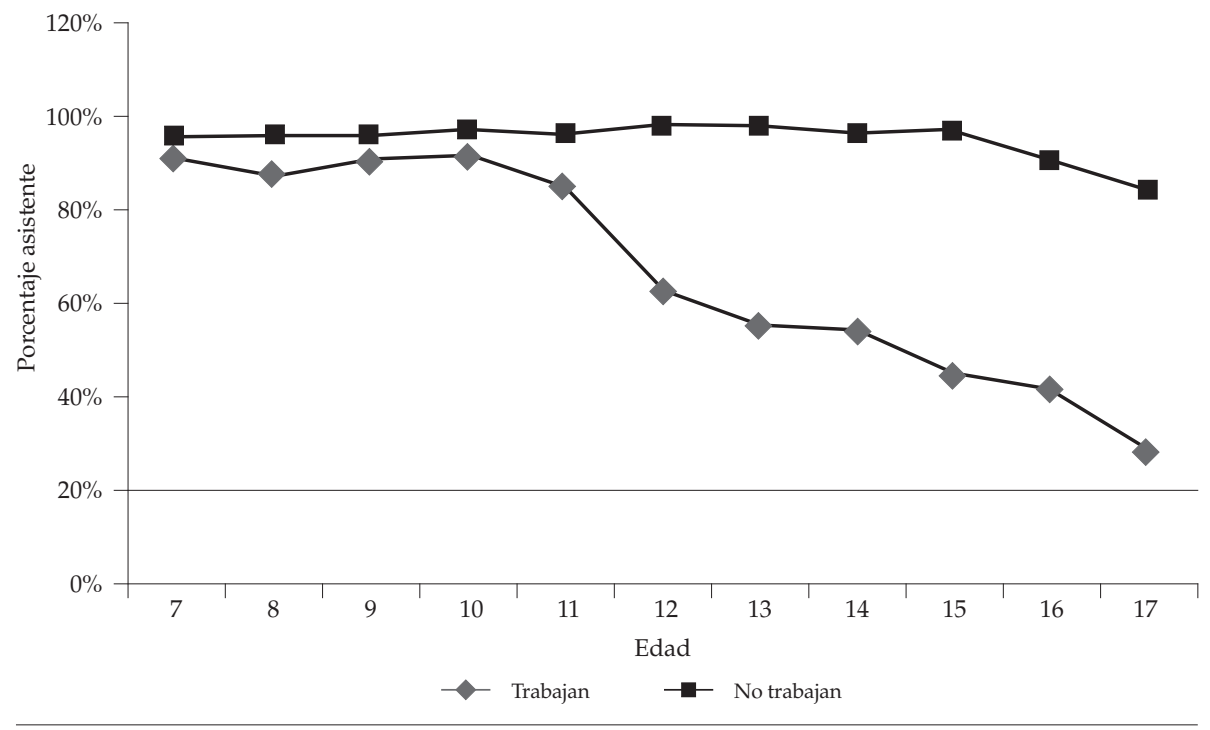

Figura 1. Asistencia escolar

Fuente: Encuesta de calidad de vida 2003.

Tabla 1. Minas ilegales por departamento

\begin{tabular}{|c|c|c|c|c|}
\hline Departamento & Pequeñas & Medianas & Grandes & Total \\
\hline Antioquia & 1183 & 412 & 5 & 1600 \\
\hline Bolívar & 888 & 445 & 9 & 1342 \\
\hline Boyacá & 692 & 145 & 8 & 845 \\
\hline Cundinamarca & 453 & 228 & 7 & 688 \\
\hline Santander & 533 & 43 & 2 & 578 \\
\hline Magdalena & 502 & 57 & 0 & 559 \\
\hline Chocó & 229 & 292 & 2 & 523 \\
\hline Cauca & 419 & 57 & 8 & 484 \\
\hline $\begin{array}{l}\text { Norte de San- } \\
\text { tander }\end{array}$ & 270 & 116 & 0 & 386 \\
\hline Putumayo & 310 & 12 & 0 & 322 \\
\hline Córdoba & 281 & 8 & 0 & 289 \\
\hline La Guajira & 186 & 91 & 0 & 277 \\
\hline Atlántico & 210 & 27 & 0 & 237 \\
\hline Valle del Cauca & 143 & 56 & 0 & 199 \\
\hline Tolima & 131 & 56 & 0 & 187 \\
\hline
\end{tabular}




\begin{tabular}{lcccc}
\hline \multicolumn{1}{c}{ Departamento } & Pequeñas & Medianas & Grandes & Total \\
\hline Caldas & 112 & 36 & 0 & 148 \\
Risaralda & 86 & 46 & 1 & 133 \\
Huila & 96 & 14 & 0 & 110 \\
Meta & 34 & 11 & 0 & 45 \\
Arauca & 12 & 13 & 1 & 26 \\
Casanare & 15 & 8 & 0 & 23 \\
Cesar & 14 & 0 & 0 & 14 \\
Caquetá & 12 & 0 & 0 & 12 \\
\hline
\end{tabular}

Fuente: Heck y Tranca (2011).

Tabla 2. Número de niños que trabajan en minería (Colombia)

\begin{tabular}{lc}
\hline \multicolumn{1}{c}{ Departamento } & Niños \\
\hline Antioquia & 51 \\
Bolívar & 35 \\
Boyacá & 12 \\
Cundinamarca & 3 \\
Santander & 6 \\
Magdalena & 5 \\
Chocó & 51 \\
Norte de Santander & 9 \\
La Guajira & 6 \\
Valle del Cauca & 1 \\
Tolima & 23 \\
Risaralda & 1 \\
\hline
\end{tabular}

Fuente: Heck y Tranca (2011).

Así, es claro que en zonas de minería artesanal el trabajo infantil es frecuente (Melguizo, 2005). ${ }^{7}$ Además, un boom aurífero, medido como una interacción entre las capacidades de producción de oro a nivel regional y los

7 La minería artesanal o minería de pequeña escala es aquella practicada por individuos, grupos o comunidades de manera informal e ilegal. Se caracteriza por las altas tasas de trabajo infantil porque las condiciones sociales y culturales bajo las cuales se desarrolla dicha labor tienden a favorecer este tipo de vinculación infantil. 
precios internacionales del oro, aumenta la probabilidad de trabajo infantil, disminuye la probabilidad de que un niño asista a la escuela y, por ende, reduce los años de educación (Santos, 2014). ${ }^{8}$

Estos hechos indican que el caso colombiano es una buena ilustración del problema que abordamos en este trabajo: cuando un sector que usa trabajo infantil se ve afectado positivamente por un choque de precios, el trabajo infantil aumenta, se reduce la escolaridad y las posibilidades de desarrollo.

\section{Modelo teórico}

Se considera un modelo de generaciones traslapadas con un consumidor representativo que vive dos períodos y cuya utilidad depende de su consumo cuando es joven $c$, de su consumo cuando es viejo $d_{t+1}$, de la educación que decida darle a sus hijos $h_{t+1}$ y del número de hijos que el hogar decida tener $N_{t+1}$.

También se supone un mercado competitivo en el sector formal donde las firmas toman los precios de los factores como dados. El salario al trabajador infantil es una función lineal del precio del bien producido.

\subsection{Hogares}

Se utiliza una función de utilidad logarítmica que combina los argumentos antes mencionados:

$$
\left.U=\left\{\log \left(c_{t}\right)+\beta\left[\log \left(d_{t+1}\right)+\gamma \log \left(h_{t+1}\right) N_{t+1}\right)\right]\right\}
$$

El número de hijos $N_{t+1}$ genera utilidad para los padres $\mathrm{y}$, en los casos en que hay trabajo infantil, además de generar utilidad, trabajan y hacen transferencias a sus padres, incrementando el ingreso familiar.

$0 \leq \beta \leq 1$ representa la paciencia de los padres o el factor de descuento. Entre mayor sea este parámetro, más pacientes son los padres.

8 Otros autores argumentan que el precio de los recursos naturales afecta la población a través de un canal institucional. Sanabria y Campo (2013) observan que para el caso colombiano la maldición de los recursos no se da por la abundancia de estos o por un aumento en sus precios, sino por el manejo institucional y el comportamiento de los agentes que intervienen en el proceso del cual se pueden generar rentas. Lo anterior dado que, aunque las regalías guarden una relación positiva con el crecimiento económico, son alicientes para la generación de corrupción y prácticas ilegales. 
En cada momento el hogar tiene un ingreso exógeno, el cual está determinado por el salario del progenitor $w_{t} h_{t}$, que depende del nivel de educación (capital humano) y un ingreso endógeno proveniente del trabajo infantil. Este ingreso depende del tiempo que los menores dedican a trabajar $\varphi_{t^{\prime}}$ del precio del recurso natural $P_{G, t}$ y de la cantidad de hijos en el hogar $\varphi_{t} P_{G, t} N_{t+1}$. Los gastos del hogar son el consumo $C_{t}$ y el costo de criar a los hijos $\varepsilon$. Por simplicidad se supone que el costo de la crianza es una función lineal del salario de los adultos $\varepsilon\left(w_{t} h_{t}\right)$. Esto puede entenderse como el costo de oportunidad de dedicar tiempo a los niños o el costo de contratar otros adultos cuyo ingreso laboral es igual al de los padres dado el supuesto de agentes homogéneos.

La primera restricción es entonces:

$$
w_{t} h_{t}+\varphi_{t} P_{G, t} N_{t+1}=C_{t}+S_{t}+\varepsilon\left(w_{t} h_{t}\right) N_{t+1}
$$

Así, el ingreso de la familia se dedica a cubrir el costo de la crianza, a consumir y a ahorrar. El consumo futuro (cuando el individuo es viejo) $d_{t+1}$ es igual al ahorro $S_{t}$ multiplicado por la tasa de interés que obtiene en el futuro $r_{t+1}$.

$$
d_{t+1}=S_{t} r_{t+1}
$$

Por último, el capital humano de los hijos es una función que depende de la productividad del sistema educativo $\delta$, de la cantidad de tiempo que el menor dedica a estudiar $\left(1-\varphi_{t}\right)$ y del nivel educativo de los padres $h_{t}$.

$$
h_{t+1}=\delta\left(1-\varphi_{t}\right) h_{t}
$$

Donde $1 \geq \varphi_{t} \geq 0$.

Para que haya producción, se requiere un nivel mínimo de capital humano de los padres, es decir, $h_{t}>0$, en adelante se adopta este supuesto. Así mismo, dado que los hogares no pueden tener infinitos hijos, se supone un número máximo de hijos igual a $\bar{N}: N_{t+1} \leq \bar{N}$.

El problema del agente representativo es maximizar la utilidad sujeto a las restricciones expresadas en las ecuaciones (2), (3) y (4). En donde las variables de decisión de cada hogar son: ahorro $\left(S_{t}\right)$, tiempo de trabajo de los niños $\left(\varphi_{t}\right)$ y número de hijos $\left(N_{t+1}\right)$.

De las condiciones de primer orden y de las restricciones de los agentes, se derivan las siguientes condiciones: 
La proporción de tiempo que los niños destinan al trabajo está dada por la siguiente expresión:

$$
\varphi_{t}=\max \left[0, \min \left(1, \frac{P_{G, t}-\beta \gamma c_{t}}{P_{G, t}}\right)\right]
$$

Así, dado el consumo, existe un nivel crítico del precio del recurso natural por debajo del cual no hay trabajo infantil.

El ahorro, bajo la solución interior, es

$$
s_{t}=\beta\left(w_{t} h_{t}+\left[\varphi_{t} P_{G, t}-\varepsilon\left(w_{t} h_{t}\right)\right] N_{t+1}\right)
$$

Combinando con la ecuación 2,

$$
\begin{aligned}
& c_{t}=(1-\beta)\left(w_{t} h_{t}+\left[\varphi_{t} P_{G, t}-\varepsilon\left(w_{t} h_{t}\right)\right] N_{t+1}\right) \\
& \text { Luego } c_{t}=\frac{1-\beta}{\beta} S_{t} .
\end{aligned}
$$

Así, el consumo presente y el ahorro son proporciones constantes del ingreso familiar neto de costos de crianza. ${ }^{9}$

Tomando la ecuación (5) y reemplazando el consumo de la ecuación (7), se obtiene:

$$
\varphi_{t}=\max \left[0, \min \left(1, \frac{P_{G, t}-\beta \gamma(1-\beta) w_{t} h_{t}\left(1-\varepsilon N_{t+1}\right)}{\left(1+\beta \gamma(1-\beta) N_{t+1}\right) P_{G, t}}\right)\right]
$$

Así, entre mayor es la relación $\frac{P_{G, t}}{w_{t} h_{t}}$ mayor es el tiempo que pasan los menores trabajando. Este resultado se explica porque el retorno de enviar los niños a trabajar depende del precio del recurso natural.

9 Este resultado que facilita sustancialmente el análisis se deriva de la función de utilidad logarítmica. 
Por otro lado, entre mayor sea la cantidad de hijos en el hogar, más fácil es que todo el tiempo de los niños se destine al trabajo. Más aún, si $N_{t+1}>\frac{1}{\varepsilon-\frac{P_{G, t}}{w_{t} h_{t}}}$, entonces $\varphi_{t}=1$.

Estos últimos resultados se explican porque, entre mayor es el número de hijos, mayor es el retorno de incrementar su tiempo de trabajo. De manera que, siempre y cuando el precio del recurso natural sea positivo, cualquier aumento en el número de hijos incrementa el retorno del tiempo de trabajo infantil.

Note también que entre más impaciente es el individuo, es decir, entre menor es $\beta$, es más probable que mande a su hijo a trabajar.

Para completar la solución del consumidor, se reemplaza el tiempo de trabajo infantil de solución interior $\varphi_{t}$ en las ecuaciones (6) y (7) y se obtienen las expresiones de consumo y ahorro para la solución interior:

$$
\begin{gathered}
S_{t}=\frac{(1-\beta)}{\left(1-(1-\beta)\left(1+\gamma N_{t+1}\right)\right)}\left(\left(w_{t} h_{t}\left(1-\varepsilon N_{t+1}\right)+P_{G, t} N_{t+1}\right)\right) \\
c_{t}=\frac{(1-\beta)}{\left(1-(1-\beta)\left(1+\gamma N_{t+1}\right)\right)}\left(w_{t} h_{t}\left(1-\varepsilon N_{t+1}\right)+P_{G, t} N_{t+1}\right)
\end{gathered}
$$

Por último, para obtener el número de hijos que el hogar decide, se parte de la condición de primer orden:

$$
\frac{\partial U}{\partial N_{t+1}}=\frac{\varphi_{t} P_{G, t}-\varepsilon\left(w_{t} h_{t}\right)}{c_{t}}+\frac{\beta \gamma}{N_{t+1}}
$$

Note que la condición de primer orden (8) no se cumple con igualdad si $\varphi_{t} P_{G, t}>\varepsilon\left(w_{t} h_{t}\right)$ y, por lo tanto, solo puede haber solución interior si $\frac{P_{G, t}}{w_{t} h_{t}}<\frac{\varepsilon}{\varphi_{t}}$. Note también que la ecuación 5' implica que si $\frac{P_{G, t}}{w_{t} h_{t}}<\beta \gamma(1-\beta)\left(1-\varepsilon N_{t+1}\right)$, entonces no hay trabajo infantil $\varphi_{t}=0$. Así, si $\varphi_{t} P_{G, t}>\varepsilon\left(w_{t} h_{t}\right)$, entonces $\varphi_{t}>0 \mathrm{y}$ $N_{t+1}=\bar{N}$. Así, si hay solución de esquina para la tasa de natalidad, entonces hay trabajo infantil.

Cuando hay trabajo infantil, hay un beneficio pecuniario de tener hijos: $\varphi_{t} P_{G, t}$. Si este es mayor al costo de tener hijos $\left(\varepsilon\left(w_{t} h_{t}\right)\right)$, el hogar siempre opta 
por aumentar el número de hijos. Ahora, como se afirmó arriba, hay un número máximo de hijos que una familia puede tener $\bar{N} .{ }^{10}$ Así, el número de hijos está dado por:

$$
N_{t+1}=\left[\begin{array}{ccc}
\min \left(\frac{\beta \gamma c_{t}}{\varepsilon\left(w_{t} h_{t}\right)-\varphi_{t} P_{G, t}}, \bar{N}\right) & \text { si } & \frac{P_{G, t}}{w_{t} h_{t}}<\frac{\varepsilon}{\varphi_{t}} \\
\bar{N} & \text { si } & P_{G, t} \geq \frac{\varepsilon}{\varphi_{t}}
\end{array}\right.
$$

Esta ecuación indica que la tasa de fertilidad depende del costo de tener hijos, de la tasa de impaciencia, de la preferencia por capital humano $\gamma$ y del posible ingreso que los hijos puedan generar. Si el ingreso aumenta, la tasa de fertilidad crece.

A partir de los anteriores resultados, es posible plantear tres casos dependiendo del número de hijos y del tiempo que los niños dedican al trabajo:

El caso en el cual los niños no trabajan y el número de hijos es el máximo posible, $\varphi_{t}=0$ y $\frac{\beta \gamma c_{t}}{\varepsilon\left(w_{t} h_{t}\right)}>\bar{N}$, no es un equilibrio porque si $\varphi_{t} P_{G, t}>\varepsilon\left(w_{t} h_{t}\right)$, entonces $\varphi_{t}>0 \mathrm{y}$, por lo tanto, si $N_{t+1}=\bar{N}$, entonces $\varphi_{t}=0$

Caso 1: $\frac{\beta \gamma c_{t}}{\left(w_{t} h_{t}\right)}<\frac{(1-\beta) \beta \gamma}{(1+(1-\beta) \beta \gamma)}$

1) Suponga que $\varphi_{t}=0$. Si $\varphi_{t}=0$, entonces $\frac{\beta \gamma c_{t}}{\left(w_{t} h_{t}\right)}<\frac{\varepsilon}{\varphi_{t}}$ y, por la ecuación (9), $N_{t+1}=\frac{\beta \gamma c_{t}}{\varepsilon\left(w_{t} h_{t}\right)}$. Así mismo, de la ecuación $\left(7^{\prime}\right)$ se sigue $c_{t}=\frac{1-\beta}{(1+(1-\beta) \beta \gamma)}$ $\left(w_{t} h_{t}\right)$ y $N_{t+1}=\frac{\beta \gamma c_{t}}{\varepsilon\left(w_{t} h_{t}\right)}=\frac{\beta \gamma}{\varepsilon} \frac{1-\beta}{(1+(1-\beta) \beta \gamma)}$.

2) $\operatorname{Si} N_{t+1}=\frac{\beta \gamma}{\varepsilon} \frac{1-\beta}{(1+(1-\beta) \beta \gamma)}$, de la ecuación (5') se sigue:

Si $\frac{P_{G, t}}{w_{t} h_{t}}<\left(\frac{(1-\beta) \beta \gamma}{(1+(1-\beta) \beta \gamma)}\right)$, entonces $\varphi_{t}=0$.

De 1) y 2) se sigue que si $\frac{P_{G, t}}{w_{t} h_{t}}<\left(\frac{(1-\beta) \beta \gamma}{(1+(1-\beta) \beta \gamma)}\right)$, entonces $\varphi_{t}=0 \mathrm{y}$ :

10 Tener en cuenta que la vida reproductiva de una mujer es limitada y, por lo tanto, existe un número limitado de hijos que se pueden tener igual a $\bar{N}$. 


$$
\begin{gathered}
s_{t}=\frac{\beta}{(1+(1-\beta) \beta \gamma)}\left(w_{t} h_{t}\right) \\
c_{t}=\frac{1-\beta}{(1+(1-\beta) \beta \gamma)}\left(w_{t} h_{t}\right) \\
N_{t+1}=\frac{\beta \gamma}{\varepsilon} \frac{(1-\beta)}{(1+(1-\beta) \beta \gamma)}
\end{gathered}
$$

En este caso, el precio del recurso natural es bajo comparado con el ingreso de los adultos de manera que no hay trabajo infantil porque el costo de oportunidad de la educación es muy bajo.

Caso 2: $\left(\varepsilon+\frac{(1-\beta) \beta \gamma}{(1+(1-\beta) \beta \gamma)}\left(1-\frac{1}{\bar{N}}\right)\right) \geq \frac{P_{G, t}}{w_{t} h_{t}}>\left(\frac{(1-\beta) \beta \gamma}{(1+(1-\beta) \beta \gamma)}\right)$

1) Del punto dos del caso anterior se sigue que si $\frac{P_{G, t}}{w_{t} h_{t}}>\left(\frac{(1-\beta) \beta \gamma}{(1+(1-\beta) \beta \gamma)}\right)$,
entonces $\varphi_{t}>0$.

2) Suponga que $\frac{\beta \gamma c_{t}}{\varepsilon\left(w_{t} h_{t}\right)-\varphi_{t} P_{G, t}} \leq \bar{N}$. De la ecuación (9) se deriva que si $\frac{P_{G, t}}{w_{t} h_{t}}<\frac{\varepsilon}{\varphi_{t}}$ y $\frac{\beta \gamma c_{t}}{\varepsilon\left(w_{t} h_{t}\right)-\varphi_{t} P_{G, t}} \leq \bar{N}$, entonces hay solución interior para el número de hijos,

$$
N_{t+1}=\frac{\beta \gamma c_{t}}{\varepsilon\left(w_{t} h_{t}\right)-\varphi_{t} P_{G, t}} \leq \bar{N}
$$

Utilizando las condiciones de primer orden y reemplazando, es posible reescribir las ecuaciones (5), (6'), (7') y (9):

$$
\begin{gathered}
\varphi_{t}=\left(1-\beta \gamma \frac{1-\beta}{(1+(1-\beta) \beta \gamma)} \frac{w_{t} h_{t}}{P_{G, t}}\right) \\
s_{t}=\frac{\beta}{(1+(1-\beta) \beta \gamma)}\left(w_{t} h_{t}\right) \\
c_{t}=\frac{1-\beta}{(1+(1-\beta) \beta \gamma)}\left(w_{t} h_{t}\right)
\end{gathered}
$$




$$
N_{t+1}=\frac{(1-\beta) \beta \gamma}{(1-\beta) \beta \gamma-\left(\frac{P_{G, t}}{w_{t} h_{t}} \varepsilon\right)(1+(1-\beta) \beta \gamma)}
$$

Note que en este caso $N_{t+1}$ es una función creciente en $\frac{P_{G, t}}{w_{t} h_{t}}$.

Note también que, dada la ecuación (7.2),

$$
N_{t+1}=\bar{N} \Leftrightarrow \frac{P_{G, t}}{w_{t} h_{t}}=\left(\varepsilon+\frac{(1-\beta) \beta \gamma}{(1+(1-\beta) \beta \gamma)}\left(1-\frac{1}{\bar{N}}\right)\right)
$$

En estas condiciones se puede observar que las formas funcionales del consumo y el ahorro son iguales a las del caso 1. Esto sucede porque los nuevos hijos generan más ingreso al hogar, pero a su vez significan un gasto adicional. Las familias escogen el número de hijos de tal forma que el costo y el ingreso se igualan.

Caso 3: $\frac{P_{G, t}}{w_{t} h_{t}}>\left(\varepsilon+\frac{(1-\beta) \beta \gamma}{(1+(1-\beta) \beta \gamma)}\left(1-\frac{1}{\bar{N}}\right)\right)$

En este caso, de las condiciones de primer orden se sigue que la familia tiene el máximo posible de hijos $\bar{N}$ y los niños trabajan.

Combinando las restricciones con las condiciones de primer orden se obtiene

$$
\begin{gathered}
c_{t}=\frac{(1-\beta)}{(1+(1-\beta) \beta \gamma \bar{N})}\left((1-\varepsilon \bar{N}) w_{t} h_{t}+P_{G, t} \bar{N}\right) \\
s_{t}=\frac{\beta}{(1+(1-\beta) \beta \gamma \bar{N})}\left((1-\varepsilon \bar{N}) w_{t} h_{t}+P_{G, t} \bar{N}\right) \\
\frac{P_{G, t}-\beta \gamma(1-\beta)(1-\varepsilon \bar{N}) w_{t} h_{t}}{P_{G, t}(1+(1-\beta) \beta \gamma \bar{N})}
\end{gathered}
$$

Recuerde que si $\bar{N} \geq \frac{1}{\left(\varepsilon \frac{P_{G, t}}{w_{t} h_{t}}\right)}$, entonces $\varphi_{t}=1$.

Ahora, si $\bar{N} \geq \overline{\left(\varepsilon \frac{P_{G, t}}{w_{t} h_{t}}\right)}$ y $\frac{P_{G, t}}{w_{t} h_{t}}>\left(\varepsilon+\frac{(1-\beta) \beta \gamma}{(1+(1-\beta) \beta \gamma)}\left(1-\frac{1}{\bar{N}}\right)\right)$, entonces 


$$
\begin{aligned}
& \frac{P_{G, t}}{w_{t} h_{t}}>\left(\varepsilon+\frac{(1-\beta) \beta \gamma}{(1+(1-\beta) \beta \gamma)}\left(1-\left(\varepsilon-\frac{P_{G, t}}{w_{t} h_{t}}\right)\right)\right), \text { reagrupando } \\
& \frac{P_{G, t}}{w_{t} h_{t}}>(\varepsilon+(1-\beta) \beta \gamma) . \\
& \text { Así, si } \frac{P_{G, t}}{w_{t} h_{t}}>(\varepsilon+(1-\beta) \beta \gamma) \text { entonces } \varphi_{t}=1 .
\end{aligned}
$$

\section{Solución general}

A partir de los casos 1, 2 y 3, es posible analizar el comportamiento de las variables.

La figura 2 muestra que la variable que determina el número de hijos es la relación entre el precio de los recursos naturales (el retorno del trabajo infantil) y el salario de los adultos (el costo de tener hijos). Dado el salario de los padres, para bajos precios de recursos naturales, la tasa de fertilidad es contante, pero a partir de un nivel crítico $\left(\frac{(1-\beta) \beta \gamma}{(1+(1-\beta) \beta \gamma)} w_{t} h_{t}\right)$ cualquier incremento en el precio de los recursos naturales genera un amento en el número de hijos. Una vez el número de hijos alcanza el máximo, deja de responder a incrementos en

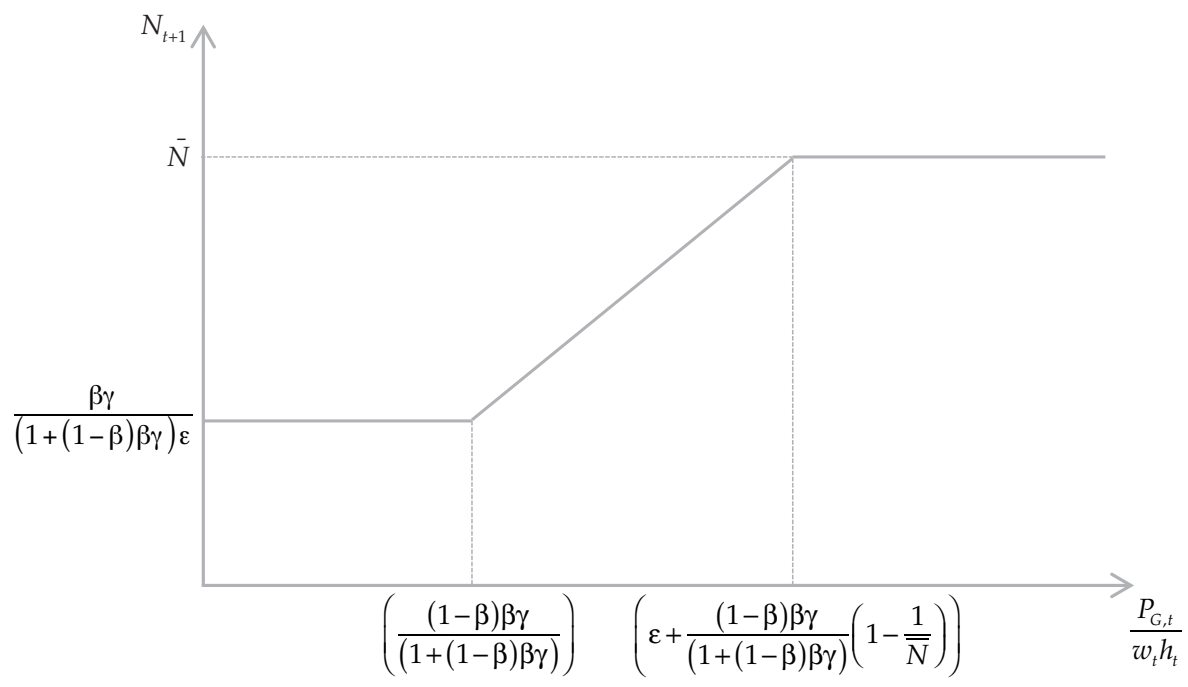

Figura 2. Precio de los recursos naturales y tasa de natalidad

Fuente: elaboración de los autores. 
el precio de los recursos naturales. Finalmente, aumentos en el salario de los adultos reducen la tasa de natalidad cuando existe solución interior.

Si el precio de los recursos naturales aumenta, los padres tienen mayores incentivos a enviar a sus hijos a trabajar porque saben que en el corto plazo su ingreso es mayor y, por lo tanto, el costo de oportunidad de enviarlos a estudiar es muy alto. Sin embargo, existe un número máximo de hijos que los hogares pueden concebir, el cual es representado por $\bar{N}$. No importa que tan alto sean los precios de los recursos naturales hay un punto en el cual la madre del hogar no puede tener más hijos.

Por su parte, la proporción del tiempo de trabajo infantil está dada por:

$$
\left\{\begin{array}{cc}
0 & \text { caso } 1 \\
\left(1-\beta \gamma \frac{1-\beta}{(1+(1-\beta) \beta \gamma)} \frac{w_{t} h_{t}}{P_{G, t}}\right) & \text { caso } 2 \\
\min \left(1, \frac{P_{G, t}-\beta \gamma(1-\varepsilon \bar{N}) w_{t} h_{t}}{P_{G, t}[(1+(1-\beta) \beta \gamma \bar{N})]}\right) & \text { caso } 3
\end{array}\right\}
$$

Combinando los tres casos, es posible describir el comportamiento del trabajo infantil. La figura 3 ilustra cómo el trabajo infantil es determinado por la relación entre el precio de los recursos naturales y el salario de los adultos. Dado el salario de los padres, para bajos precios de recursos naturales, no hay trabajo infantil, pero a partir de un nivel crítico $\left(\frac{(1-\beta) \beta \gamma}{(1+(1-\beta) \beta \gamma)} w_{t} h_{t}\right)$ cualquier incremento en el precio de los recursos naturales genera un amento en el trabajo infantil.

Si el precio del recurso natural es relativamente alto comparado con el salario de los adultos, hay trabajo infantil y este es creciente en el precio de los recursos naturales. Finalmente, cuando todo el tiempo de los niños está dedicado al trabajo, aumentos en el precio de los recursos naturales no tienen efecto. 


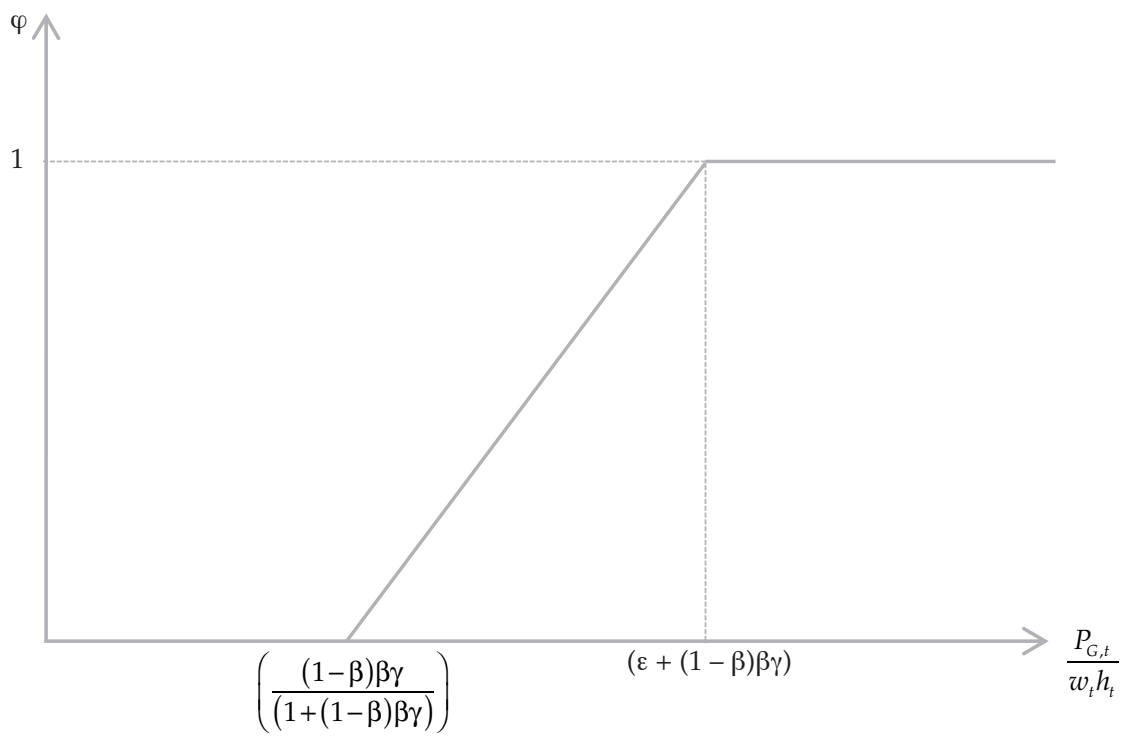

Figura 3. Precio de los recursos naturales y trabajo infantil Fuente: elaboración de los autores.

Dada la función de utilidad logarítmica, el consumo es una proporción constante de los ingresos del hogar,

$$
\begin{array}{cc}
c_{t}=\left\{\begin{array}{cc}
\frac{(1-\beta)}{(1+(1-\beta) \beta \gamma)}\left(w_{t} h_{t}\right) & \text { casos 1y2 } \\
\frac{(1-\beta)}{(1+(1-\beta) \beta \gamma \bar{N})}\left((1-\varepsilon \bar{N}) w_{t} h_{t}+P_{G, t} \bar{N}\right) & \text { caso3 }
\end{array}\right. \\
s_{t}=\left\{\begin{array}{cc}
\frac{(1-\beta)}{(1+(1-\beta) \beta \gamma)}\left(w_{t} h_{t}\right) & \text { casos 1y2 } \\
\frac{(1-\beta)}{(1+(1-\beta) \beta \gamma \bar{N})}\left((1-\varepsilon \bar{N}) w_{t} h_{t}+P_{G, t} \bar{N}\right) & \text { caso3 }
\end{array}\right.
\end{array}
$$

Cuando el precio del recurso natural es relativamente bajo y no hay trabajo infantil, todo el ingreso del hogar viene del trabajo de los adultos. Si el precio del recurso natural es relativamente alto y hay trabajo infantil, el hogar tiene ingresos más altos, pero también mayores costos, puesto que el número de hijos es mayor. Bajo la solución interior estos dos efectos se cancelan. 
No obstante, cuando hay solución de esquina para el número de hijos, el efecto positivo del aumento en el precio del recurso natural es mayor.

\subsection{Firmas y precios de los factores}

Se supone una función de producción Cobb-Douglas para los bienes finales.

$$
Y_{t}=A K_{t}^{\alpha} H_{t}^{1-\alpha}=A K_{t}^{\alpha}\left(h_{t} L_{t}\right)^{1-\alpha}
$$

Donde $Y$ es el producto, $K$ el capital físico agregado, $H$ el capital humano agregado, $L$ el número de trabajadores.

De manera que el ingreso por trabajador es

$$
Y_{t}=A K_{t}^{\alpha} h_{t}^{1-\alpha}
$$

Siendo $k$ la relación capital-trabajo.

La ecuación (14) representa la cantidad producida $Y$ utilizando como factores de producción el capital humano $H$ y el capital físico $K$. $A$ es una constante positiva que representa la tecnología, $\alpha$ y $\alpha-1$ son menores que uno y representan el peso de los factores $H$ y $K$ en la distribución de la renta.

El número de trabajadores en $t$ está definido como: $L_{t}=L_{t-1} N_{t}$, en donde $L_{t-1}$ es el número de adultos en $t-1$ y $N_{t}$ es el número de hijos. Por lo tanto, el capital humano es:

$$
H_{t}=h_{t} L_{t}
$$

Los precios de los factores se determinan de manera competitiva suponiendo la función de producción de bienes finales.

Los beneficios de la empresa son

$$
\pi=A k_{t}^{\alpha} h_{t}^{1-\alpha}-w_{t} h_{t}-r_{t} k_{t}
$$

Así, las condiciones de primer orden de la firma son

$$
r=\alpha A k^{\alpha-1} h^{1-\alpha} ; \mathrm{w}=(1-\alpha) A k^{\alpha} h^{-\alpha}
$$

Así, los precios de los factores son, en equilibrio, iguales a sus productividades marginales. 


\subsection{Condiciones de equilibrio y dinámica del capital}

La oferta de capital humano por individuo es igual a la demanda de las firmas:

$$
h_{t}=\left(\frac{(1-\alpha) A}{w_{t}}\right)^{\frac{1}{\alpha}} k_{t}=\delta\left(1-\varphi_{t-1}\right) h_{t-1} .
$$

La oferta de activos es igual al ahorro individual $S_{t}$ por el número de individuos $L_{t}$. Las firmas demandan activos para hacerlos capital. Así,

$$
K_{t-1}=s_{t} L_{t}
$$

El capital por trabajador es, entonces, igual a: $\frac{K_{t+1}}{L_{t+1}}=\frac{K_{t+1}}{L_{t} N_{t+1}}=\frac{s_{t} L_{t}}{N_{t} N_{t+1}}=\frac{s_{t}}{N_{t+1}}$

Para encontrar el ahorro individual, se combinan las ecuaciones (12) y (16):

$S_{t}=\left\{\begin{array}{cc}\frac{\beta}{(1+(1-\beta) \beta \gamma)}\left((1-\alpha) A k^{\alpha} h^{1-\alpha}\right) & \text { casos } 1 y 2 \\ \frac{(\beta)}{(1+(1-\beta) \beta \gamma \bar{N})}\left((1-\varepsilon \bar{N})(1-\alpha) A k^{\alpha} h^{1-\alpha}+P_{G, t} \bar{N}\right) & \text { caso3 }\end{array}\right.$

Utilizando la ecuación $K_{t+1}=\frac{s_{t}}{N_{t+1}}$, se obtiene:

$$
\frac{k_{t+1}}{k_{t}}=\left\{\begin{array}{cc}
\frac{\beta}{N_{t+1}}\left[\frac{(1-\alpha) A k^{\alpha} h^{1-\alpha}}{(1+(1-\beta) \beta \gamma)}\right] & \text { casos } 1 y 2 \\
\frac{\beta}{\bar{N}}\left[\frac{\left((1-\varepsilon \bar{N})(1-\alpha) A k^{\alpha} h^{1-\alpha}+P_{G, t} \bar{N}\right)}{(1+(1-\beta) \beta \gamma \bar{N})}\right] & \text { caso3 }
\end{array}\right.
$$

Recuerde que en el caso 3 el hogar tiene un número máximo de hijos, por eso en este caso $k_{t+1}=\frac{s_{t}}{\bar{N}}$

Para encontrar la tasa de crecimiento del capital humano $h$, se usan las ecuaciones (3), (10) y (17), 


$$
\frac{h_{t+1}}{h_{t}}\left\{\begin{array}{cc}
\delta & \text { caso } 1 \\
\frac{\beta \gamma(1-\beta)(1-\alpha) A k^{\alpha} h^{1-\alpha}}{(1+(1-\beta) \beta \gamma) P_{G, t}} \delta & \text { caso } 2 \\
\frac{\left\{(1-\beta)(1-\varepsilon \bar{N})(1-\alpha) A k^{\alpha} h^{1-\alpha} P_{G, t} \bar{N}\right\}}{P_{G, t}[(1+(1-\beta) \beta \gamma \bar{N})]} & \\
\beta \gamma \delta & \text { caso3 }
\end{array}\right.
$$

Cuando no hay trabajo infantil, la acumulación de capital humano depende únicamente de la productividad de este sector $h_{t+1}=\delta h_{t}$. Esto solo sucede cuando el precio de los recursos naturales es bajo porque, una vez este aumente, los incentivos de los padres siempre serán enviar a sus hijos a trabajar en las minas.

\subsection{Resultados}

Proposición 1: Si $\delta>1$ y $\frac{P_{G, t}}{w_{t} h_{t}} \leq\left(\frac{(1-\beta) \beta \gamma}{(1+(1-\beta) \beta \gamma)}\right)$, entonces para todo t existe una senda de crecimiento balanceado, $\frac{k_{t+1}}{k_{t}}=\frac{h_{t+1}}{h_{t}}=\delta y \frac{K}{h}=\left(\frac{\varepsilon(1-\alpha) A}{\delta(1-\beta) \gamma}\right)^{\frac{1}{1-\alpha}}$.

[La demostración está en el apéndice].

La proposición 1 indica que si el precio de los recursos naturales es relativamente bajo y la productividad del sistema educativo es alta, los padres no tienen incentivos de mandar a sus hijos a trabajar y, por esto, el capital humano tiene una senda creciente. En estas condiciones hay crecimiento del capital humano, del capital físico y del producto.

Ahora, note que si $\frac{P_{G, t}}{w_{t} h_{t}}>\left(\frac{(1-\beta) \beta \gamma}{(1+(1-\beta) \beta \gamma)}\right)$, entonces la tasa de crecimiento del capital humano depende negativamente del precio del recurso natural.

$$
h_{t+1}=\left\{\begin{array}{cc}
\beta \gamma \delta h_{t} \frac{\left\{(1-\beta)\left[(1-\varepsilon \bar{N})(1-\alpha) A k^{\alpha} h^{1-\alpha}+P_{G, t}\right\}\right.}{P_{G, t}[(1+(1-\beta) \beta \gamma \bar{N})]} & \text { si } P_{G, t}>\left(\frac{(1-\beta) \beta \gamma}{(1+(1-\beta) \beta \gamma)}\right) w_{t} h_{t} \\
\delta h_{t} & \text { si } P_{G, t} \leq\left(\frac{(1-\beta) \beta \gamma}{(1+(1-\beta) \beta \gamma)}\right) w_{t} h_{t}
\end{array}\right.
$$


Así, si $\frac{P_{G, t}}{w_{t} h_{t}}>\left(\frac{(1-\beta) \beta \gamma}{(1+(1-\beta) \beta \gamma)}\right)$, entonces la tasa de crecimiento del capital humano es negativa.

Este resultado implica que aumentos en el precio de los recursos naturales pueden generar caídas en la acumulación de capital humano.

Proposición 2: Si $N=\bar{N} y \frac{P_{G, t}}{w_{t} h_{t}}>\frac{\beta \gamma(1-\beta)(1-\varepsilon \bar{N})}{1-\left(\frac{\delta-1}{\delta}\right)[(1+(1-\beta) \beta \gamma \bar{N})]}$, entonces siempre que haya trabajo infantil la tasa de crecimiento del capital humano es negativa.

[La demostración está en el apéndice].

La proposición 2 indica que por encima de cierto nivel del precio de los recursos naturales el tiempo de asistencia escolar es tan bajo que el capital humano decrece.

Proposición 3: SiP ${ }_{G, t}$ es constante, entonces, para cualquier condición inicial tal que $\frac{1}{\Omega}>\frac{P_{G, t}}{w_{t} h_{t}}>\left(\varepsilon+\frac{(1-\beta) \beta \gamma}{(1+(1-\beta) \beta \gamma)}\right)$, existe un estado estacionario caracterizado por:

$$
k^{*}=\frac{\beta \varepsilon\left(P_{G, t} * \Omega\right)-\frac{\delta-1}{\delta}\left(\frac{1}{\Omega}\right)}{\delta \beta \gamma(1-\beta)\left((1-\varepsilon \bar{N})+\frac{1 \bar{N}}{\Omega}\right)} y h^{*}=\left(\frac{P_{G, t}}{\Omega(1-\alpha) A k^{* \alpha}}\right)^{\frac{1}{1-\alpha}} .
$$

Donde $\Omega=\frac{1-\frac{\delta-1}{\delta}[(1+(1-\beta) \beta \gamma \bar{N})]}{\beta \gamma(1-\beta)(1-\varepsilon \bar{N})}$.

[La demostración está en el apéndice].

La proposición 3 indica que si el precio del recurso natural es suficientemente alto para que haya trabajo infantil y es persistentemente alto, entonces en el largo plazo el capital humano no crece y, por lo tanto, la economía se estanca.

Proposición 4: Si $\left(\varepsilon+\left(\frac{(1-\beta) \beta \gamma}{(1+(1-\beta) \beta \gamma)}\right)-\frac{(1-\beta) \beta \gamma}{w_{t} h_{t}}\right)<\frac{P_{G, t}}{w_{t} h_{t}}<(\varepsilon+$. $\left.\frac{(1-\beta) \beta \gamma}{(1+(1-\beta) \beta \gamma)}\left(1-\frac{1}{\bar{N}}\right)\right)$, entonces la tasa de crecimiento del capital físico es negativa.

[La demostración está en el apéndice].

En este caso, el precio de los recursos naturales es tan alto comparado con el ingreso de los adultos que hay trabajo infantil y alta fertilidad, pero no es suficientemente grande para que el ingreso del hogar permita aumentos 
sustanciales en el ahorro y la acumulación de capital físico. Así, el efecto del número de hijos compensa el aumento en capital haciendo que el capital por trabajador se reduzca.

\subsection{Implicaciones de politica}

Los resultados de la sección anterior indican que la relación entre el ingreso de los padres y el ingreso que derivan los menores de su trabajo es la variable central en las decisiones de la familia. Esto implica que, todo lo demás constante, el nivel de los salarios de los adultos es determinante. Un mayor salario para los adultos puede reducir o eliminar el trabajo infantil. El problema aquí es que en las regiones más pobres el salario promedio suele ser inferior al salario mínimo legal de manera que la mayoría del trabajo es informal. Así, el reto de política es impulsar el desarrollo de empresas formales en estas zonas.

En segundo lugar, lo que en nuestro modelo es la productividad del sector educativo incluye, en la práctica, dos elementos: la capacidad de educar de las escuelas y el retorno a la educación. La literatura acerca de los mecanismos que permiten mejorar el nivel de las escuelas es extensa, pero casi toda concuerda en la necesidad de invertir en este sector. Con respecto al retorno a la educación, en la medida en que haya un sector formal capaz que demande mano de obra calificada, el retorno a la educación será alto. Así, estimular la creación de empresas formales en estas zonas puede atacar el problema del trabajo infantil por dos vías.

En este orden de ideas, el otorgamiento de incentivos tributarios a las firmas que inviertan y contraten población nativa en regiones donde el trabajo infantil es común puede ayudar a reducir este fenómeno.

Del mismo modo, otorgar condiciones laborales especiales a los maestros que enseñan en regiones afectadas por trabajo infantil puede ayudar a incrementar la calidad de la educación en estas regiones.

En lo que respecta al problema de incentivos, un programa de transferencias condicionadas que garantice una suma de dinero a las familias que envían sus hijos a estudiar reduce el costo de oportunidad de enviar a los menores al colegio. En Colombia, Familias en Acción es un programa de estas características que ha sido exitoso en varias dimensiones. No obstante, en zonas de minería tradicional sigue habiendo trabajo infantil, de manera que valdría la pena estudiar formas de ajustar el programa para las zonas de minería artesanal. En particular, las transferencias condicionadas pueden ser más cuantiosas en las zonas de minería tradicional.

Por último, y apartándonos un poco de las implicaciones del modelo, para estimular a los niños a acudir al colegio es deseable invertir en instalaciones 
deportivas y actividades extracurriculares que incrementen la satisfacción derivada de acudir al colegio y articular los programas de vivienda social con los programas educativos para reducir costos de desplazamiento de los estudiantes.

\section{Conclusiones}

Este artículo presenta un análisis teórico de la 'maldición de los recursos naturales' desde la perspectiva de las decisiones del hogar. La principal pregunta es cómo el precio de los recursos naturales afecta las decisiones del hogar sobre el número de hijos del hogar y la asignación de tiempo de los niños entre la educación y el trabajo. Estas decisiones afectan a su vez la tasa de crecimiento del capital humano y la senda de crecimiento de la economía.

En equilibrio, para las familias de bajos ingresos, los niños son fuente importante de ingresos para el hogar y, por lo tanto, el costo de oportunidad de la educación es muy alto. En el caso de las familias de altos ingresos, el potencial aporte de los niños al ingreso familiar es pequeño y, por esto, la utilidad que derivan los padres de la educación de los hijos es mayor al costo de oportunidad de enviarlos a estudiar.

Las decisiones de los padres afectan el ingreso de la familia en el corto plazo y los salarios futuros de los menores. Así, la decisión de enviar a los niños a trabajar hace que los salarios futuros sean bajos y, por lo tanto, perpetúa las condiciones de pobreza.

Cuando el precio de los recursos naturales es alto comparado con el ingreso del hogar, el aporte relativo de los niños al ingreso familiar se hace más alto y, por lo tanto, aumenta el trabajo infantil y pueden surgir trampas de pobreza. Además, el incremento en los ingresos laborales de los menores estimula la tasa de natalidad y puede reducir la relación capital-trabajo de la siguiente generación.

Del análisis teórico se derivan tres implicaciones de política: 1) Aumentar la productividad en la educación puede ayudar a reducir el trabajo infantil y contribuye a eliminar trampas de pobreza. 2) Ante un incremento en los precios de los recursos naturales se debe reforzar la política social. Es decir, parte de la bonanza debe destinarse a reducir el costo de oportunidad de ir a estudiar. 3) Instrumentos de política social, como las transferencias condicionadas, pueden ser útiles para romper las trampas de pobreza que se originan en las zonas de minería. 


\section{Referencias}

Becker, G., \& Tomes, N. (1994). Human capital and the rise and fall of families. Human capital: a theoretical and empirical analysis with special reference to education ( $3^{\text {rd }}$ ed.). The University of Chicago Press.

Campo, J., \& Sanabria, A. (2013). Recursos naturales y crecimiento económico en Colombia: ¿maldición de los recursos? En Perfil de coyuntura económica (pp. 17-37). Universidad de Antioquia. Recuperado de http://www. redalyc.org/pdf/861/86130434002.pdf

Cogneau, D., \& Jedwab, R. (2007). Household income and investments in child health and education in Ivory Coast. Journal of Economic Literature, 40.

Departamento Administrativo Nacional de Estadística (DANE). (2003). Encuesta de calidad de vida 2003 (ECV 2003). Bogotá: DANE.

Doepke, M. (2004). Accounting for fertility decline during the transition to growth. Journal of Economic Growth, 9(3), 347.

Edmonds, E. V., \& Pavcnik, N. (2004). Child labor in the global economy. Journal of Economic Perspectives, 19(1), 199-220.

Galor, O. (2005). The demographic transition and the emergence of sustained economic growth. Journal of European Economic Association, 3, 494-504.

Galor, O., \& Moav, O. (2004). From physical to human capital accumulation: inequality in the process of development. Review of Economic Studies, 71, 1001-1026.

Galor, O., \& Weil, D. (1999). From Malthusian stagnation to modern growth. American Economic Review, 89(2), 150-154.

Galor, O., \& Zang, H. (1997). Fertility, income distribution, and economic growth: theory and cross-country evidence. Japan and the World Economy, 9(2), 197-229.

Gómez, D. F., Giraldo, J., Ramírez, C., \& Aparicio, C. (2013). Factores condicionantes del trabajo infantil en las ciudades de Bogotá, Cali, Medellín, Barranquilla, Cartagena e Ibagué. Análisis cuantitativo y cualitativo. Fundación Telefónica. Recuperado de http:/ /www.fundaciontelefonica.co/wpcontent/ uploads/2015/08/Investigacion_FTC_ECSIM.pdf

Heck, C., \& Tranca, J. (2011). La realidad de la minería ilegal en países amazónicos. Sociedad Peruana de Derecho Ambiental. Recuperado de http:/ / www.actualidadambiental.pe/wp-content/uploads/2014/06/La-realidad-de-la-miner\%C3\%ADa-ilegal-en-pa\%C3\%ADses-amaz\%C3\%B3nicosSPDA.pdf

Heckman, J. J., \& Raut, L. K. (2013). Intergenerational long term effects of preschoolstructural estimates from a discrete dynamic programming model. Nber Wor- 
king Paper Series, National Bureau of Economic Research. Recuperado de http:/ / www.nber.org/papers/w19077

Ishikawa, T. (1975). Family structures and family values in the theory of income distribution. Journal of Political Economy, 83(5), 987-1008.

Kraay, A., \& McKenzie, D. (2014). Do poverty traps exist? Assessing the evidence. Journal of Economic Perspectives, 28(3), 127-148.

Márcio, E., Kassouf, A. L., \& Arends-Kuenning, M. (2009). The impact of child labor and school quality on academic achievement in Brazil. Discussion Paper, 4062. Recuperado de http:/ /ftp.iza.org/dp4062.pdf

Parsons, D., \& Goldin, C. (1981). Economic well-being and child labor: the interaction of family and industry. National Bureau of Office and Research, Working Paper 707. Recuperado de http:/ / www.nber.org/papers/w0707.pdf

Patrinos, H. A., \& Psacharopoulos, G. (1997). Family size, schooling and child labor in Peru. An empirical analysis. Journal of Population Economics, 10(4), 387-406.

Pinzón-Rondón, A. M., Briceño-Ayala, L., Botero, J. C., Cabrera, P., \& Rodríguez, M. N. (2006). Trabajo infantil ambulante en las capitales latinoamericanas. Salud Pública de México, 48(5), 363-372.

Rosati, F. C., \& Rossi, M. C. (2003). Children's working hours and school enrollment: evidence from Pakistan and Nicaragua. The World Bank Economic Review, 17(2), 283-295.

Santos, R. J. (2014). Not all that glitters is gold: gold boom, child labor and schooling in Colombia. Documento CEDE 31.

Van der Ploeg, F. (2011). Natural resources: curse or blessing? Journal of Economic Literature, 49(2), 366-420. 


\section{Apéndice}

Proposición 1: Si $\delta>1$ y $\frac{P_{G, t}}{w_{t} h_{t}} \leq\left(\frac{(1-\beta) \beta \gamma}{(1+(1-\beta) \beta \gamma)}\right)$ para todo t, entonces existe un patrón de crecimiento balanceado, $\frac{k_{t+1}}{k_{t}}=\frac{h_{t+1}}{h_{t}}=\delta y \frac{K}{h}=\left(\frac{\varepsilon(1-\alpha) A}{\delta(1-\beta) \gamma}\right)^{\frac{1}{1-\alpha}}$.

Demostración:

En este caso, los niños no trabajan luego $\frac{h_{t+1}}{h_{t}}=\delta$.

De la ecuación (18) se sigue que si $P_{G, t}<\left(\frac{(1-\beta) \beta \gamma}{(1+(1-\beta) \beta \gamma)}\right) w_{t} h_{t}$, entonces $\frac{k_{t+1}}{k_{t}}=\frac{\beta w_{t} h_{t}}{N_{t-1}(1+(1-\beta) \beta \gamma) k_{t}}$

Teniendo en cuenta que $N_{t-1}=\frac{\beta \lambda c_{t}}{\varepsilon\left(w_{t} h_{t}\right)-\varphi_{t} P_{G, t}}$, $\frac{k_{t+1}}{k_{t}}=\frac{\beta w_{t} h_{t} \varepsilon\left(w_{t} h_{t}\right)}{\beta \gamma c_{t}(1+(1-\beta) \beta \gamma) k_{t}}$

Igualando $\frac{k_{t+1}}{k_{t}}=\frac{h_{t+1}}{h_{t}}$ se obtiene,

$\frac{\beta w_{t} h_{t} \varepsilon\left(w_{t} h_{t}\right)}{\beta \gamma c_{t}(1+(1-\beta) \beta \gamma) k_{t}}=\delta$

Reemplazando $C_{t}$ de la ecuación (7.2), $w_{t} h_{t}$ de la ecuación (16) y reagrupando, $\frac{k}{h}=\left(\frac{\varepsilon(1-\alpha) A}{\delta(1-\beta) \gamma}\right)^{\frac{1}{1-\alpha}}$

Proposición 2: $S i \frac{P_{G, t}}{w_{t} h_{t}}>\frac{\beta \gamma(1-\beta)(1-\varepsilon \bar{N})}{1-\frac{\delta-1}{\delta}(1+(1-\beta) \beta \gamma \bar{N})}$, entonces siempre que haya trabajo infantil la tasa de crecimiento del capital humano es negativa.

(i) $\frac{h_{t+1}}{h_{t}}=\delta(1-\varphi)$ luego si $\varphi_{t}>\frac{\delta-1}{\delta}$, entonces $\frac{h_{t+1}}{h_{t}}<1$. 
(ii) De la ecuación (10) se sigue que $\varphi_{t}=\frac{P_{G, t}-\beta \gamma(1-\beta)(1-\varepsilon \bar{N}) w_{t} h_{t}}{P_{G, t}[(1+(1-\beta) \beta \gamma \bar{N})]}$, luego $\frac{P_{G, t}-\beta \gamma(1-\beta)(1-\varepsilon \bar{N}) w_{t} h_{t}}{P_{G, t}[(1+(1-\beta) \beta \gamma \bar{N})]}>\frac{\delta-1}{\delta} \rightarrow \varphi_{t}>\frac{\delta-1}{\delta}$. Reagrupando, Si $\frac{P_{G, t}}{w_{t} h_{t}}>\frac{\beta \gamma(1-\beta)(1-\varepsilon \bar{N})}{1-\left(\frac{\delta-1}{\delta}\right)[(1+(1-\beta) \beta \gamma \bar{N})]}$, entonces $\varphi_{t}>\frac{\delta-1}{\delta}$. $\frac{h_{t+1}}{h_{t}}<1$.

De (i) y (ii) se sigue que si $\frac{P_{G, t}}{w_{t} h_{t}}>\frac{\beta \gamma(1-\beta)(1-\varepsilon \bar{N})}{1-\left(\frac{\delta-1}{\delta}\right)[(1+(1-\beta) \beta \gamma \bar{N})]}$, entonces
$<1$.

Proposición 3: $S i P_{G, t}$ es constante, entonces, para cualquier condición inicial tal que $\frac{P_{G, t}}{w_{t} h_{t}}>\left(\varepsilon+\frac{(1-\beta) \beta \gamma}{(1+(1-\beta) \beta \gamma)}\right)$, existe un estado estacionario caracterizado por

$$
k^{*}=\frac{\beta \varepsilon\left(P_{G, t} * \Omega\right)-\frac{\delta-1}{\delta}\left(\frac{1}{\Omega}\right)}{\delta \beta \gamma(1-\beta)\left((1-\varepsilon \bar{N})+\frac{1 \bar{N}}{\Omega}\right)} y h^{*}=\left(\frac{P_{G, t}}{\Omega(1-\alpha) A k^{* \alpha}}\right)^{\frac{1}{1-\alpha}} .
$$

Donde $\Omega=\frac{1-\frac{\delta-1}{\delta}[(1+(1-\beta) \beta \gamma \bar{N})]}{\beta \gamma(1-\beta)(1-\varepsilon \bar{N})}$.

\section{Demostración:}

(i) Igualando $\frac{k_{t+1}}{k_{t}}=\frac{h_{t+1}}{h_{t}}$ se obtiene $k_{t}^{*}=\frac{\beta w_{t} h_{t} \varepsilon\left(w_{t} h_{t}\right)-\varphi_{t} P_{G, t}}{\delta \beta \gamma c_{t}(1+(1-\beta) \beta \gamma)}$

(ii) $\frac{h_{t+1}}{h_{t}}=\left(1-\varphi_{t}\right) \delta$, luego si $\frac{h_{t+1}}{h_{t}}=1$, entonces $\varphi_{t}=\frac{\delta-1}{\delta}$.

(iii) $\frac{P_{G, t}}{w_{t} h_{t}}>\left(\varepsilon+\frac{(1-\beta) \beta \gamma}{(1+(1-\beta) \beta \gamma)}\right)$ implica que estamos en el caso 3, donde

$c_{t}=\frac{(1-\beta)\left((1-\varepsilon \bar{N}) w_{t} h_{t}+P_{G, t} \bar{N}\right)}{(1+(1-\beta) \beta \gamma \bar{N})}$

(iv) $\varphi_{t}=\frac{\delta-1}{\delta}=\frac{P_{G, t}-\beta \gamma(1-\beta)(1-\varepsilon \bar{N}) w_{t} h_{t}}{P_{G, t}[(1+(1-\beta) \beta \gamma \bar{N})]}$. Dividiendo arriba y abajo entre $P_{G, t t}$ 


$$
\frac{\delta-1}{\delta}=\frac{1-\beta \gamma(1-\beta)(1-\varepsilon \bar{N}) \frac{w_{t} h_{t}}{P_{G, t}}}{(1+(1-\beta) \beta \gamma \bar{N})} \operatorname{luego} \frac{w_{t} h_{t}}{P_{G, t}}=\frac{1-\frac{\delta-1}{\delta}[(1+(1-\beta) \beta \gamma \bar{N})]}{\beta \gamma(1-\beta)(1-\varepsilon \bar{N})} \equiv \Omega .
$$

De (i), (ii) y (iii) se deduce que si existe un estado estacionario, entonces la relación capital-trabajo de largo plazo está dada por

$$
k_{t}^{*}=\frac{\beta \varepsilon\left(w_{t} h_{t}\right)-\frac{\delta-1 P_{G, t}}{\delta P_{G, t}}}{\delta \beta \gamma(1-\beta)\left((1-\varepsilon \bar{N}) \frac{1 \bar{N}}{\Omega}\right)} \frac{(1+(1-\beta) \beta \gamma \bar{N})}{(1+(1-\beta) \beta \gamma)}
$$

De (iv) se deduce que $\left(w_{t} h_{t}\right)$ es igual a $P_{G, t}{ }^{*} \Omega$. Así, dado $P_{G, t}, k_{t}^{*}$ tiene solución única y hay un estado estacionario único.

$$
k_{t}^{*}=\frac{\beta \varepsilon\left(P_{G, t}^{*} \Omega\right)-\frac{\delta-1}{\delta}\left(\frac{1}{\Omega}\right)}{\delta \beta \gamma(1-\beta)\left((1-\varepsilon \bar{N}) \frac{1 \bar{N}}{\Omega}\right)} \frac{(1+(1-\beta) \beta \gamma \bar{N})}{(1+(1-\beta) \beta \gamma)}
$$

Para encontrar $h^{*}$ partimos de $\Omega=\frac{P_{G, t}}{w_{t} h_{t}}$.

Reemplazando $w_{t} h_{t}$ de la ecuación (16), $\Omega=\frac{P_{G, t}}{(1-\alpha) A k^{\alpha} h^{1-\alpha}}$.

Despejando $h$,

$$
\begin{aligned}
& h^{*}=\left(\frac{P_{G, t}}{\Omega(1-\alpha) A k^{* \alpha}}\right)^{\frac{1}{1-\alpha}} . \\
& \frac{P_{G, t}}{w_{t} h_{t}}=\left(\varepsilon+\frac{(1-\beta) \beta \gamma}{(1+(1-\beta) \beta \gamma)}\left(1-\frac{1}{\bar{N}}\right)\right)
\end{aligned}
$$

Proposición 4: $S i\left(\varepsilon+\left(\frac{(1-\beta) \beta \gamma}{(1+(1-\beta) \beta \gamma)}\right)-\frac{(1-\beta) \gamma}{w_{t} h_{t}}\right)<\frac{P_{G, t}}{w_{t} h_{t}}<(\varepsilon+$ $\left.\frac{(1-\beta) \beta \gamma}{(1+(1-\beta) \beta \gamma)}\left(1-\frac{1}{\bar{N}}\right)\right)$, entonces la tasa de crecimiento del capital físico es negativa. 
Demostración:

(i) $\operatorname{Si} \frac{P_{G, t}}{w_{t} h_{t}}<\left(\varepsilon+\frac{(1-\beta) \beta \gamma}{(1+(1-\beta) \beta \gamma)}\left(1-\frac{1}{\bar{N}}\right)\right)$, entonces hay solución interior para $\mathrm{N}_{\mathrm{t}+1}$.

(ii) De las ecuaciones (9.2) y (18) se deduce

$$
\begin{aligned}
& \frac{k_{t+1}}{k_{t}}=\left(\varepsilon-\frac{P_{G, t}}{w_{t} h_{t}}+\beta \gamma \frac{(1-\beta)}{(1+(1-\beta) \beta \gamma)}\right)\left[\frac{(1-\alpha) A k^{\alpha-1} h^{1-\alpha}}{(1-\beta) \gamma}\right] . \\
& \text { Así, si }\left(\varepsilon-\frac{P_{G, t}}{w_{t} h_{t}}+\beta \gamma \frac{(1-\beta)}{(1+(1-\beta) \beta \gamma)}\right)\left[\frac{(1-\alpha) A k^{\alpha-1} h^{1-\alpha}}{(1-\beta) \gamma}\right]<1, \text { entonces } \frac{k_{t+1}}{k_{t}}<1 .
\end{aligned}
$$

Reordenando, si $\left(\left(\frac{(1-\beta) \gamma \beta}{(1+(1-\beta) \beta \gamma)}-\varepsilon\right)-\frac{(1-\beta) \gamma}{w_{t} h_{t}}\right)<\frac{P_{G, t}}{w_{t} h_{t}}$, entonces $\frac{k_{t+1}}{k_{t}}<1$.

De (i) y (ii) se sigue que si $\left(\left(\frac{(1-\beta) \gamma \beta}{(1+(1-\beta) \beta \gamma)}+\varepsilon\right)-\frac{(1-\beta) \gamma}{w_{t} h_{t}}\right)<\frac{P_{G, t}}{w_{t} h_{t}}<$ $\varepsilon+\frac{\beta \gamma(1-\beta)}{1+\beta \gamma(1-\beta)}$, entonces la tasa de crecimiento del capital físico es negativa. 
